\title{
61. Is Aging of Tumor Cells Related to the Alteration of Stemline Karyotypes ?'
}

\author{
By Tosihide H. Yosida \\ National Institute of Genetics, Misima \\ (Comm. by Hitoshi Kinara, M. J. A., April 12, 1972)
}

Based on the extensive cytogenetical studies of the Yoshida rat sarcoma, Makino $(1952,1957)$ has established a concept of tumor stemline cells for the transplantable tumors. According to him, each tumor has a cell lineage with characteristic chromosome constitution dividing regularly through cell generations. Levan and Hauschka (1952) used a term of germ-line in almost the same meaning as the stemline. A concept of the stemline in the transplantable tumors has been accepted by many cytogeneticists.

According to Makino (1952) the most important character of tumor stemline cells is constancy of the stemline idiogram through many transfer generations. The constancy, however, is not always absolute as Makino (1957) later recognized it himself. Change of tumor stemline karyotypes has been studied by many investigators in several tumors. Hauschka and Levan (1958) emphasized in tumor progression which is facilitated by continuous selective population shift within a randomly mutating multiple stemline system. Based on the cytogenetical studies of various experimental tumors the present author (Yosida 1966, 1968) explained the karyotype alteration of tumor stemline cells to depend on a sequential event of mutation and selection of tumor cells. Here is a question why transplantable tumors have a stemline consisting of a certain karyotype through many generations, in spite of the continuous and random occurrence of mutations in the cell population. Can we explain this phenomenon by only sequential events of mutation and selection?

To consolidate the different opinion on the behaviour of tumor stemline cells, constancy and variability, I would like to propose the idea of the "aging" of the tumor cells. All multicellular or unicellular organisms and sexual or unsexual reproducing organisms have the age. In the sexual reproducing organisms, the life will be extended by the recombination following the mating of sexually different

1) Contribution No. 879 from the National Institute of Genetics, Japan. Supported by grant-in-aid for the fundamental scientific research from the Ministry of Education, Japan (92332). 
individuals. Mating and recombination do not occur in the somatic cells, except the hybridization of in vitro culturing cells. In human diploid cell strain, the diploid karyological character is limited to about 50 serial passages which correspond to about one year in vitro culture (Hayflick and Moorhead 1961). Thereafter the mitotic activity decreases and aneuploidy appears in the cell population (Saksela and Moorhead 1963). Among the aneuploid cells a modal karyotype is observed as the stemline of the permanent cell line, but the stemline karyotype does not continue for so long generations, but also are replaced by the other karyotypic cells. In the human cell line, the life span of about 50 passages seems to be the age of the diploid cell line. An aneuploid cell line arisen after the diploid cell degeneration is the second stem-cell population. The karyotype of the second stemline cells, however, is replaced by the tertiary stem-cell population after a certain period by the same mechanism as above. Thus, the proliferation of the re-established cell line should be limited by "aging" as the first diploid cells.

In karyotype alteration in the tumor stemline cells could be explained by the same mechanism as the above. Only a difference between the tumor strain and cultured lines is that the tumor strain growing in vivo will receive a more strict selection by immune responses than the cultured line growing in vitro. Therefore, a karyotype of the tumor stemline is stable for a long period as Makino demonstrated. But, there is a limitation on the stability of the tumor stemline karyotype by aging. Life span or age of the tumor stemline seems to be different by strain of tumor. For instance, in the MY mouse sarcoma drastic change of karyotype from diploidy to tetraploidy was observed after about the 90th transplant generation, which corresponds to about 6 years. In Yoshida rat sarcoma, the karyotype change was observed sometimes during the tumor transplantations (Yosida 1959, Matsushima and Yosida 1971). A rapid karyotype alteration has been observed in mouse plasma cell tumors. In this case the karyotype change from diploidy to tetraploidy occurred within one to two transplant generations (Yosida et al. 1970). In the rat chloroma (Shay) the karyotype alteration occurred sequentially with a period of about 2 years. In this case, karyotype alteration occurred by sequential change of chromosome breakages, translocation and also inversion (Yosida and Sakai unpublished). Thus, the karyotype alteration of tumor stemline cell occurs sequencially one to another (Fig. 1). Stemline karyotype A shown in the figure degerates after a certain transfer generations by the age, and the new cells with karyotype $B$, which occurred by chomosomal alteration from the stemline $\mathrm{A}$, increase in the cell population, and 


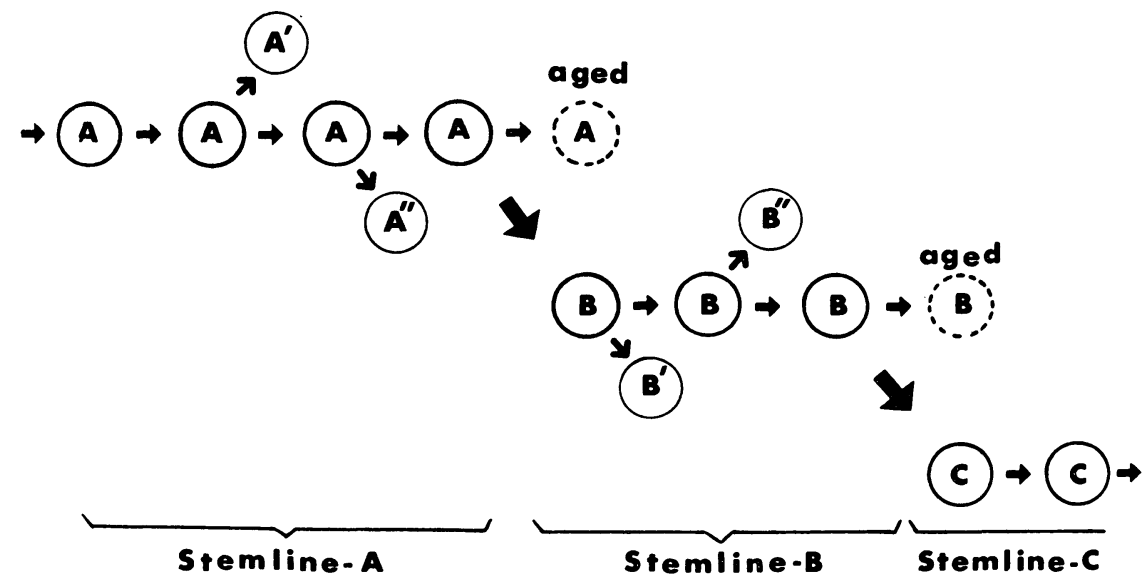

Fig. 1. Process of alteration of stemline karyotypes by aging of stem cells. $\mathrm{A}, \mathrm{B}$ and $\mathrm{C}$ mean the karyotypes of stemline cells. After degeneration of the stemline cell A by aging, the stemline B develops from the stemline $\mathrm{A}$ by chromosome alteration, and the stemline $\mathrm{C}$ from the stemline $\mathrm{B}$ by the same mechanism as above. $\mathrm{A}^{\prime}, \mathrm{A}^{\prime \prime}, \mathrm{B}^{\prime}$ and $\mathrm{B}^{\prime \prime}$ mean the altered cells from the stemline $A$ and $B$ cells, but they can not proliferate over the stemline cells.

the tumor is occupied by the new stemline $B$. The stemline $B$ is replaced again by stemline $\mathrm{C}$ after a certain period as the same mechanism as the above.

The karyotype change in the tumor stemline is facilitated when the tumor was put in the hostile condition. Gross karyotype change occurred in a mouse lymphocytic leukemia P388 when the tumor cells were treated with 8-azaguanine and amethopterine (Yosida 1966) and 5-fluorodeoxyuridine (Yosida 1968). A large marker chromosome which was usually observed in the stemline cells of the mouse leukemia L1210 disappeared when the tumor was treated with amethopterin, 6-mercaptopurine and 5-fluorouracil (Yosida 1967). These instances could be explained by that the age of the tumor stem cells is shortened by treatment with the above drugs and the stemline is replaced by the new cell type.

Here is a question why the tumor stemline has the limited age. Why the tumor stemline cells degenerate after a certain period of the cell multiplication? An unfavorable gene or genes should be accumulated in the stemline cells during the progress of transplant generations and the chromosome alteration should be necessary to change the function of the aged cells. Functional change of the cells following the ploidy shift or chromosome structural change in the tumor stemline has been demonstrated in several tumor strains. The change in enzyme activity in the ploidy shift was demonstrated 
in Ehrlich ascites tumor (Kid and Gross 1959, Tsukada et al. 1959), and in cultured hepatic cells (Konigsberg et al. 1962). Quantitative change of protein synthesis following the karyotype shift has been demonstrated in the Yoshida sarcoma and mouse plasma cell tumors (Moriwaki et al. 1969). Change of infiltration ability into organs has been demonstrated in the karyological mutants of the Yoshida rat sarcoma and Ehrlich mouse tumor (Yosida 1959, 1964). Change of cell surface antigens following karyotype alteration has been considered by Hauschka et al. (1956), Sacks and Gallily (1956) and Amos (1956).

Does the functional change of tumor cells by the chromosome alteration reflect the revival of cell lineage? Unfavorable genes accumulated in the cell should not be detrimental to the mutated cells having functions differing from the parent one. The gross change of stemline karyotype has been demonstrated in the resistant cell lines which developed after repetitious treatments with some chemicals (Yosida 1966, 1967, 1968). Mutant cell lines thus established are not only resistant to the chemicals, but sometimes require such chemicals to maintain the cell life as a dependent mutant. In the light of above instances, it can be said that the change in gene function following the chromosomal alteration might play an important role in the revival of the aged stemline cells.

Conclusion and summary: From the several studies on chromosome alteration in tumor stem cells a concept on aging of tumor cells was postulated to explain the continuity of cell lineage. Tumor stemline cells seemed to be degenerated by aging after a certain transfer generation caused by accumulation of unfavorable gene or genes. The aged cells are revived by the functional change resulted from the karyotype alteration. When the stemline cells have reached the aged stage, a certain cell with altered karyotype, which has the strongest competitive ability among the mutants, occupies the cell population as the second stemline cell. After a certain period, the second stemline cells become aged stage, and a new revived cell type occurs in the cell population as the third stemline. Thus, the tumor stemline evolves by karyotype alteration and the transplantable tumor continues its life through many cell generations.

The above concept has been reported in 1971 Annual Meeting of the Society of Chromosome Research in Japan (Yosida and Sakai) and by Matsushima and Yosida (1971), and the details of the study was reported in the International Symposium on "Recent results in cancer research" to be held in Düsseldorf in March, 1972. 


\section{References}

Amos, D. B.: Serological differences between comparable diploid and tetraploid lines of three mouse ascites tumors. Ann. New York Acad. Sci., 63, 706-710 (1956).

Hauschka, T. S., Kvedar, B. J., Grinell, S. T. et al.: Immunoselection of polyploids from predominantly diploid cell populations. Ann. New York Acad. Sci., 63, 683-705 (1956).

Hauschka, T. S., and Levan, A.: Cytological and functional characterization of single cell clones isolated from the Krebs-2 and Ehrlich ascites tumors. J. Nat. Cancer Inst., 21, 77-135 (1958).

Hayflick, L., and Moorhead, P. S.: The serial cultivation of human diploid cell strain. Exp. Cell Res., 25, 585-621 (1961).

Kid, S., and Gross, A. L.: Quantitative relationships between DNA content and glycolysis or histones of diploid and tetraploid cells. Biochem. Biophys. Acta, 36, 185-191 (1959).

Konisberg, U. R., and Nitowsky, H. M.: Studies of the karyotype of clonal strains of Chang liver differing in alkaline-phosphatase activity. J. Nat. Cancer Inst., 29, 699-709 (1962).

Levan, A., and Hauschka, T. S.: Chromosome numbers of three mouse ascites tumors. Hereditas, 38, 251-255 (1952).

Makino, S.: A cytological study of the Yoshida sarcoma, an ascites tumor of white rats. Chromosoma (Berl.), 4, 649-674 (1952).

- : The chromosome cytology of the ascites tumors of rats, with special reference to the concept of stemline cells. Intern. Rev. Cytol., 6, 26-84 (1957).

Matsushima, T., and Yosida, T. H.: Chromosomal atleration and the development of tumors. XXII. Change of stemline karyotype in Yoshida sarcoma by appearance of peculiar marker chromosomes. Gann, 62, 389-394 (1971).

Moriwaki, K., Imai, H. T., and Yosida, T. H.: Polyploidyzation and protein synthesis in mammalian tumor cells. Jap. J. Genet., 44, Suppl. 1, 71-83 (1969).

Sacks, L., and Gallily, R.: The chromosomes and transplantability of tumors. II. Chromosome duplication and the loss of strain specificity in solid tumors. J. Nat. Cancer Inst., 16, 803-841 (1956).

Saksela, E., and Moorhead, P. S.: Aneuploidy in the degenerative phase of serial caltivation of human cell strains. Proc. Nat. Acad. Sci., 50, 390-395. (1963).

Tsukada, H., and Fu, S. C. J.: Correlation between malignancy and respiratory metabolism of three sublines of Ehrlich ascites tumor cells. Proc. Ann. Cancer Res., 3, 70 (1959).

Yosida, T. H.: Chromosomal alteration and the development of tumors. I. A new subline of Yoshida sarcoma developed by chromosomal mutation. Zeit. Krebsforsch., 63, 209-214 (1959).

- : Chromosome alteration and the development of tumors. XV. Change of chromosome pattern in 8-azaguanin and amethopterin resistant sublines of the mouse lymphocytic neopasm, P388, cultured in vitro. Jap. Jour. Genet., 41, 59-74 (1966).

- Relation between chromosomal alteration and development of tumors. Jap. Jour. Genet., 41, 439-451 (1966).

- Relation between chromosomal alteration and development of tumors in vivo and in vitro. Cancer Cell in Culture (ed. by H. Katsuta), Univ. Tokyo Press, Tokyo, 171-194 (1968). 
Yosida, T. H., Ohara, K., and Law, L. W.: Chromosomal alteration and the development of tumors. XVI. Karyological studies on sensitive and resistant sublines to several antitumor agents. Jap. J. Genet., 42, 338-347 (1967).

Yosida, T. H., Ohara, H., and Roosa, R. A.: Chromosomal alteration and the development of tumors. XVIII. Karyotype of a 5-fluorodeoxyuridine resistant subline in the mouse lymphocytic neoplasma, P388, growing in vitro. Jap. J. Genet., 43, 49-56 (1968).

Yosida, T. H., Imai, H. T., and Moriwaki, K.: Chromosomal alteration and development of tumors. XXI. Cytogenetic studies of primary plasma-cell neopasms induced in BALB/c mice. J. Nat. Cancer Inst., 45, 411-418 (1970). 